\title{
Design of the Position Controller for the Leaves of MLC Based on Anti-windup
}

\author{
Xin Zhang ${ }^{1, a^{*}}$, Jianwu Dang ${ }^{2, b}$ and Yangping Wang $^{3, c}$ \\ (School of Automation \& Electrical Engineering, Lanzhou Jiaotong University, Lanzhou 730070, \\ China.) \\ azhangx@mail.Izjut.cn, bjwdang@mail.Izjtu.cn, cypwang@mail.Izjtu.cn
}

\begin{abstract}
Keywords: Particle swarm optimization; Fractional order Anti-windup; Parameter optimization; Radiation therapy; Multi-leaf collimator
\end{abstract}

\begin{abstract}
According to requirements of the leaves position precision control of multi-leaf collimator (MLC) in the process of radiotherapy, the structure and working principle of the multi-leaf collimator of conformal radiation therapy apparatus was described. The motor that drives the leaf of the MLC was taken as the control object. Firstly, the module of the motor has been established, and then the PSO algorithm was used for the parameter optimization of fractional order Anti-windup controller, in order to ensure the position accuracy of the MLC leaves and meet the requirements of conformal therapy. The experimental results show that this method can meet the needs of the control precision of the leaves' position of the multi-leaf collimator, the control effect, especially in the aspect of anti-interference is better than the traditional PID controller.
\end{abstract}

\section{Introduction}

With the deeply research on radiotherapy, the development of equipment for radiotherapy has made great progress. Then it is very important to precisely locate on the tumor and adjust the radiation dose. The purpose of the Multi-leaf Collimator( MLC) is to realize the conformal radiotherapy[1,2] So, the research of the position $[3,4]$ of the leaves of the MLC has much more practical significance[5].

In the motor speed control system, there is a phenomenon of windup. Scholars proposed some anti-windup control technology[6], which is divided into two categories: the conditional integral method and the trace back calculation method, in order to overcome the phenomenon of windup.

Particle Swarm Optimization [7](PSO) is an evolutionary computation technique that has been successfully used for many years in different domain. In this paper, the parameters of fractional order anti-windup are optimized based on PSO for the leaves position controller of MLC and the simulation gives an ideal result.

\section{Control System Model of the Multi-leaf Collimator}

The multi-leaf collimator is mainly composed by a plurality of leaves, which are arranged in pairs. In radiation therapy system, the position of MLC leaves will be determined based on the shape of the tumor, and the shape of the rays that pass through the MLC is similar to the projection on the irradiated surface of the tumor. The working principle diagram of MLC is shown in Fig.1.

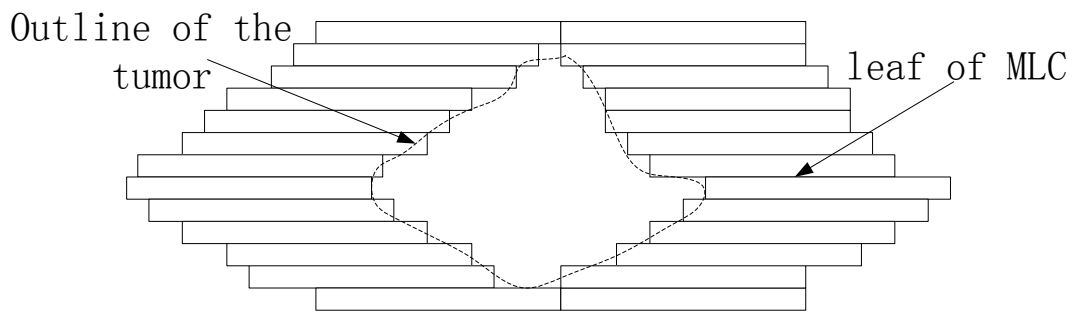

Figure 1. working principle diagram of MLC 
In practical applications, each leaf of MLC is driven by an micro electric motor, and the rotational movement of the motor is converted to a linear motion of the leaf by screw rod. Each leaf has a set of independent controller. The electric motor that has been used in this system includes stepping motor, DC servo motor, and brushless DC motor. The brushless DC motor (BLDC motor) is widely used in MLC control system with many unique advantages [8]. The structure diagram of the close-loop position control system is shown in Fig. 2.

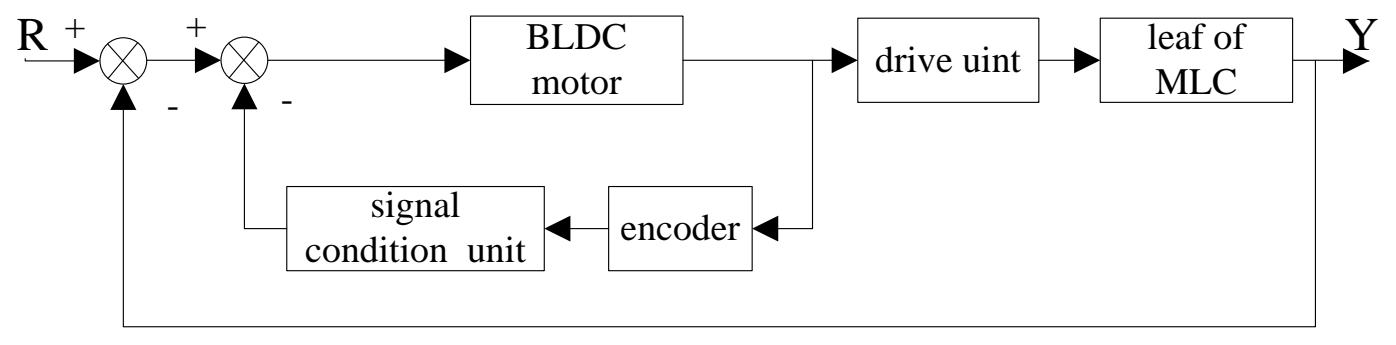

Figure 2. Structure diagram of the close-loop position control system

The paper has made much research only on the control of the motor. The BLDC motor can be controlled precisely by the drive file of MLC outputted from the radiation treatment planning system. The characteristic equations of BLDC motors can be represented as follow $[9,10]$ :

$$
\begin{aligned}
& v_{\text {app }}(t)=L \frac{d i(t)}{d t}+R i(t)+v_{\text {emf }}(t) \\
& v_{\text {emf }}=K_{\mathrm{b}} \omega(t) \\
& T(t)=K_{\mathrm{t}} i(t) \\
& T(t)=J \frac{d \omega(t)}{d t}+D \omega(t)
\end{aligned}
$$

where $v_{\text {app }}$ is the applied voltage, $\omega(t)$ is the motor speed, $L$ is the inductance of the stator, $i(t)$ is the current of the circuit, $R$ is the resistance of the stator, $v_{\text {emf }}$ is the back electromotive force, $T$ is the torque of motor, $D$ is the viscous coefficient, $J$ is the moment of inertia, $K_{\mathrm{t}}$ is the motor torque constant, and $K_{\mathrm{b}}$ is the back electromotive force constant. From the characteristic equations of BLDC motor, the transfer function of speed model is obtained as Eq.5.

$$
G(s)=\frac{Y(s)}{R(s)}=\frac{K_{t}}{L J s^{2}+(L D+R J) s+K_{\mathrm{t}} K_{\mathrm{b}}}
$$

The parameters of the motor used for simulation are as follows: $K_{\mathrm{b}}$ is the motor torque constant: $3.08 \times 10^{-3} \mathrm{~N} \cdot \mathrm{m} / \mathrm{A} ; K_{\mathrm{b}}$ is the back electromotive force constant: $0.0294 ; L$ is the inductance of the stator: $0.035 \mathrm{mH} ; J$ is the moment of inertia: $0.75 \mathrm{gcm}^{2} ; R$ is the resistance of the stator: $1.26 \Omega ; D$ is the viscous coefficient: $7.14 \times 10^{-5}$.

\section{Design of FOAnti-windup Controller}

In this paper, combined with anti-windup and fractional order PID as the controller of the controlled object, the state transition condition of the controller is realized by the judgment of the integral state q. It can be represented as Eq.6, the Structure diagram of FOAnti-windup control system is shown 
in Fig.3.

$$
q^{\prime}=\left\{\begin{array}{cc}
K_{i} e(t) & u_{n}=u_{s} \\
-\frac{1}{\tau} q & u_{n}>u_{s}
\end{array}\right.
$$

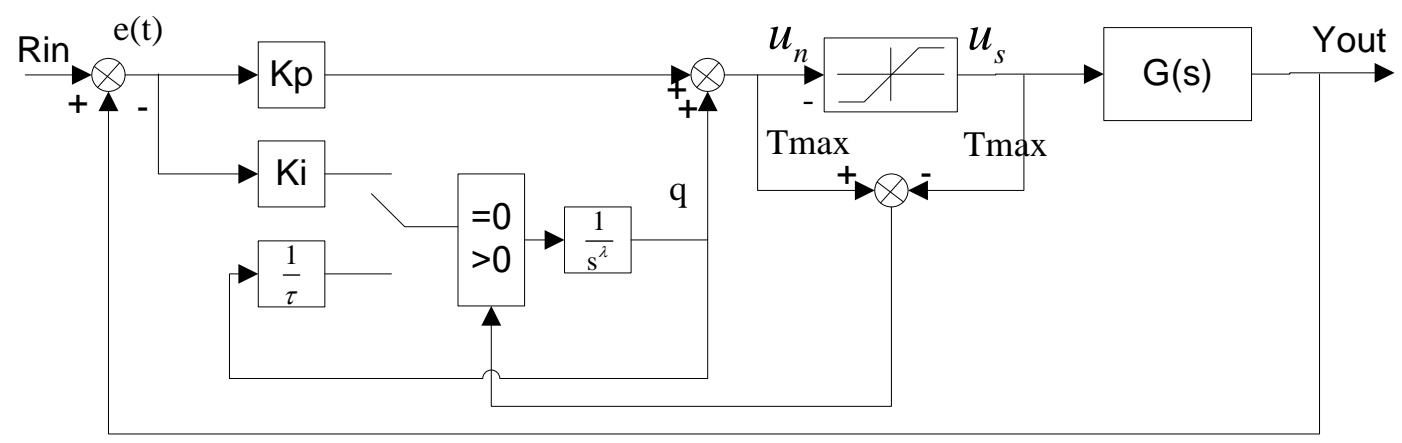

Figure 3. Structure diagram of FOAnti-windup control system

The structure diagram of FOAnti-windup control system based on PSO is shown in Fig.4.

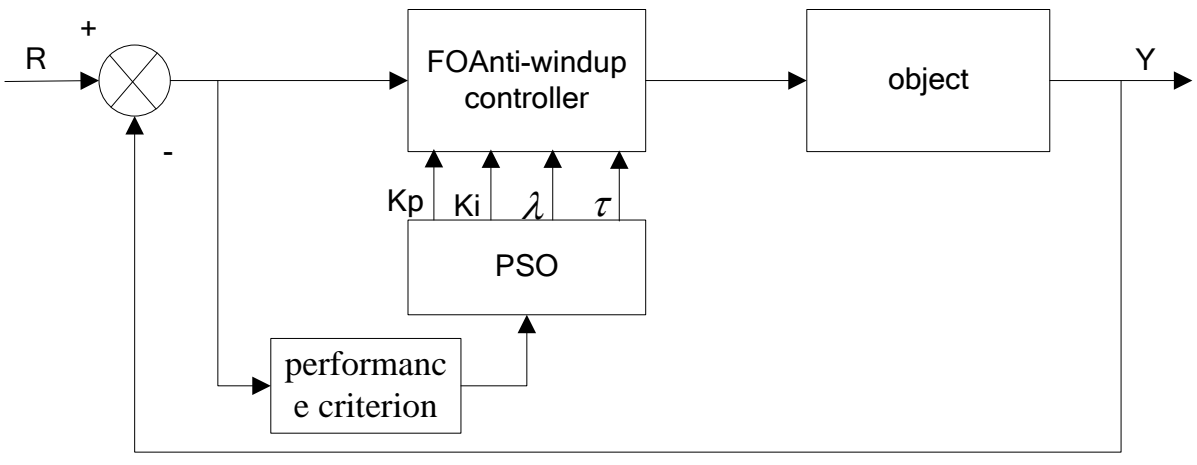

Figure 4. Structure diagram of FOAnti-windup control system based on PSO

This controller is designed mainly for the following two components: the PID controller for the object and the module of the PSO algorithm.

According to the operating state of the system, the module of PSO can optimize the parameters of the PID controller to meet the performance requirements, and the output of this module will provide the optimized parameter of PID controller.

The IATE: $J=\int_{0}^{\infty}|e(t) t d t|$ is selected as the performance criteria of this PSO- FOAnti-windup controller in the paper.

\section{Simulation and Results}

The design takes the step signal as input signal to achieve the step response of the control system. Another two examples of the fuzzy adaptive PID and PSO-PID controller is given to illustrate the proposed design is effective. The results of the three methods are shown in Fig.5. The results of the simulation show that the performance index of the proposed method is better than that by using the fuzzy adaptive PID algorithm and the PSO-PID.

\section{Conclusions}

Particle Swarm algorithm is a robust, simple and very efficient optimization algorithm. In this paper, 
the PSO FOAnti-windup controller is designed for the motor of the MLC. The paper explains how to optimize the parameter of Anti-windup controller based on the PSO algorithm. The research and simulation results show that the proposed method can obtain the satisfactory control effects; the method is versatile, effective and feasible. The result indicates that the motor can stably run according to the MLC drive file that is outputted by the radiation treatment planning system, so that the leaves can reach the assigned position accurately.

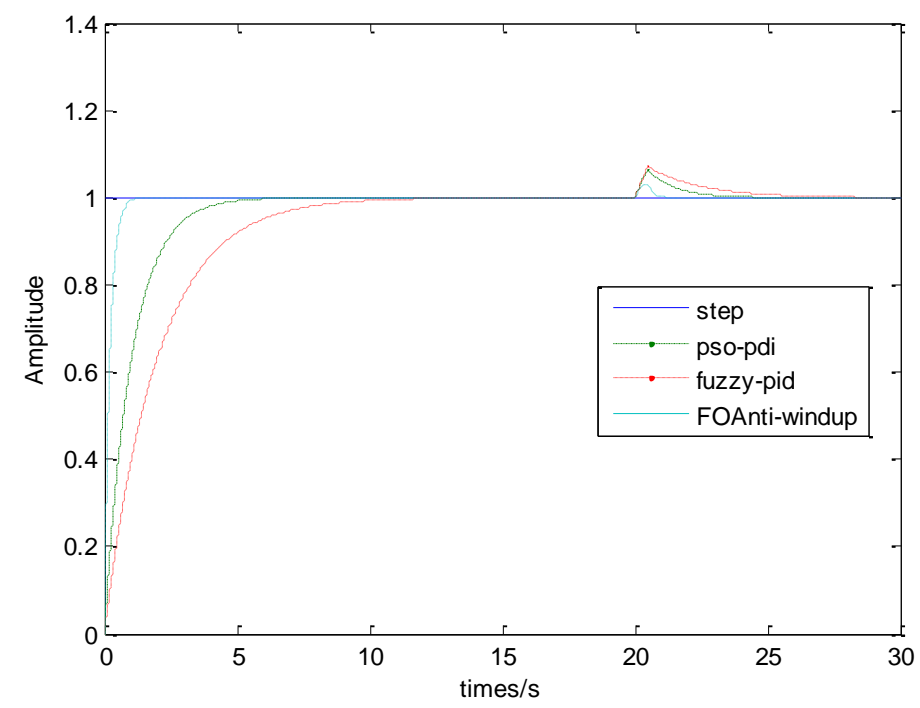

Figure 5. Step response curve of output with the three control strategies

\section{Acknowledgements}

The authors would like to thank for Project Supported by National Natural Science Foundation of China (Grant No. 61162016、60962004) and the Lanzhou Jiaotong University Youth Foundation (No. 2013041)

\section{References}

[1] J.W.Dang, Y.P.Wang, S.Li, and Z.P.Zhu. Heavy-ion Radiotherapy Treatment Planning System and Medical Image Processing Algorithm Used in It. 4th IEEE Conference on Automation Science and Engineering. Key Bridge Marriott, Washington DC. (2008),p.726.

[2] Y.P.Wang: Research on Key Technology of Image Processing for Heavy-ion 3D Conformal Radiotherapy. (Ph.D., Lanzhou: Lanzhou Jiaotong University, China 2010. (In Chinese)

[3] X.Zhang, J.W.Dang, Y.P.Wang. Parameters Optimization Based on PSO for PID controller of MLC. Journal of Lanzhou Jiaotong University. Vol.32(2013)No.6， p.15.(In Chinese)

[4] I.Podlubny, I.Petras, B.M.Vinagre, Y.Q.Chen, P.O'Leary, L.Dorcak.Realization of fractional order controllers [J]. Acta MontanisticaSlovaca (S1335-1788), Vol.8(2003) No.4,p. 233.

[5] Z.J.Ju, Y.L.Wang, L.Ma, S.P.Xu, X.K.Dai, L.Y.Wang. Verification of accuracy of multileaf collimator leaf position using a two- dimensional ion chamber array. Chinese Journal of Radiation Oncology. Vol.15(2006) No.4P.335. (In Chinese)

[6] Y.J.Yu, F.Chai, H.W.Gao, S,K.Cheng. Design of PMSM System Based on Anti-Windup Controller. Transactions of China Electrotechnical Society. Vol.24(2009) No.4P.66. (In Chinese)

[7] Kennedy, R. Eberhart. Proceedings of the IEEE International Conference on Neural Networks(Piscataway, New Jersey,1995),p.1942. 
[8] G.X.Zhu: Research on Several Key Techniques of Clinic of Multi-leaf Collimator Radiation Therapy System. (Ph.D., Shenyang University of Technology, China 2008. (In Chinese)

[9] M.O.Chee:Dynamic Simulation of Electric Machinery(Prentice Hall, New Jersey,1998).

[10]M.Piotr. Pelczewski and U.H.Kunz. The optimal control of a constrained drive system with brushless dc motor. IEEE Transactions on Industrial Electronics. Vol.37 (1990)No.5,p .342. 